\title{
FOXO1-mediated epigenetic modifications are involved in the insulin-mediated repression of hepatocyte aquaporin 9 expression
}

\author{
LIE-WANG QIU ${ }^{1 *}$, LU-YUN GU ${ }^{2 *}$, LIN LÜ ${ }^{2}$, XIAO-FENG CHEN ${ }^{2}$, CHUAN-FEI LI ${ }^{2}$ and ZHE-CHUAN MEI ${ }^{2}$ \\ ${ }^{1}$ Department of Gastroenterology, The Affiliated Yongchuan Hospital, Chongqing Medical University, Chongqing 402160; \\ ${ }^{2}$ Department of Gastroenterology, The Second Affiliated Hospital, Chongqing Medical University, \\ Chongqing 400010, P.R. China
}

Received November 29, 2013; Accepted July 1, 2014

DOI: $10.3892 / \mathrm{mmr} .2014 .3085$

\begin{abstract}
Aquaporin (AQP) 9 transports glycerol and water, and belongs to the aquaglyceroporin subfamily. Insulin acts as a negative regulator of $\mathrm{AQP9}$, and FOXO1 has the ability to mediate the regulatory effects of insulin on target gene expression. The aim of the present study was to determine whether insulin-induced repression of AQP9 involved an epigenetic mechanism. HepG2 human hepatocyte cells were treated with $500 \mu \mathrm{M}$ insulin for different durations. AQP9 mRNA expression levels were determined by quantitative polymerase chain reaction (qPCR), and histone $\mathrm{H} 3$ acetylation, phosphorylation and methylation at the insulin responsive element (IRE) of the $A Q P 9$ promoter was assessed using chromatin immunoprecipitation coupled with qPCR. The effects of lentiviral FOXO1 overexpression on AQP9 expression levels and $\mathrm{H} 3$ modifications at the $A Q P 9$ promoter were also determined. The insulin treatment resulted in a significant and time-dependent reduction in AQP9 mRNA expression levels in HepG2 cells, as compared with untreated cells $(\mathrm{P}<0.05)$. In the insulin-treated cells, the levels of $\mathrm{H} 3$ acetylation and phosphorylation were significantly reduced $(\mathrm{P}<0.05)$, but the level of $\mathrm{H} 3$ methylation was increased. Enforced expression of FOXO1 increased AQP9 mRNA and protein expression levels in HepG2 cells. Furthermore, FOXO1 overexpression promoted $\mathrm{H} 3$ acetylation and phosphorylation, and reduced $\mathrm{H} 3$ methylation at the IRE locus of the $A Q P 9$ promoter. These data provide, to the best of our knowledge, the first evidence that insulin-induced transcriptional suppression of
\end{abstract}

Correspondence to: Professor Zhe-Chuan Mei, Department of Gastroenterology, The Second Affiliated Hospital, Chongqing Medical University, 74 Linjiang Road, Yuzhong, Chongqing 400010, P.R. China

E-mail: meizhechuan@21cn.com

${ }^{*}$ Contributed equally

Key words: chromatin remodeling, gene regulation, histone, metabolic syndrome, post-transcriptional modifications
AQP9 expression in hepatocytes involves FOXO1-mediated H3 modifications at the IRE locus in the promoter.

\section{Introduction}

Aquaporins (AQPs) are a family of ubiquitous membrane proteins that form pores for the selective permeation of water and other small molecules (1). Aquaglyceroporins belong to a subgroup of the AQP family and are able to transport small organic compounds, such as glycerol or urea. Overall, five molecules (AQP3, AQP7, AQP9, AQP10 and bacterial glycerol facilitator) have been classified as aquaglyceroporins (2). AQP9 is most abundantly expressed in the liver (3). Rojek et al (4) reported that AQP9 knockout mice exhibit hypertriacylglycerolemia, a sign of metabolic syndrome. AQP9 is implicated in hepatic glycerol transport and consequently contributes to neoglucogenesis (5). Therefore, the dysregulation of AQP9 gene expression is important in the pathogenesis of metabolic disorders.

Compelling evidence has indicated that insulin acts as a key regulator of $\mathrm{AQP} 9(6,7)$. The $A Q P 9$ promoter contains a negative insulin response element (IRE), TGTTTTC, at -496/-502 and AQP9 mRNA expression is downregulated by insulin in cultured hepatocytes (6). Rodríguez et al (8) observed that insulin inhibited the expression of AQP9 via the PI3K/Akt/mTOR signaling pathway. In a rat model, hepatic AQP9 expression levels were found to fluctuate with circulating insulin levels (9). Forkhead box protein $\mathrm{O} 1$ (FOXO1) is a forkhead transcriptional factor that mediates the regulatory effects of insulin on target gene expression $(10,11)$. Tsuchida et al (12) reported that insulin negatively modulates the expression levels of adiponectin receptors via the PI3K/FOXO1-dependent pathway. A previous study demonstrated that FOXO1 was implicated in the regulation of AQP9 expression, as depletion of FOXO1 using small interfering RNA technology was observed to reduce the transcriptional activation of AQP9 (13). These findings suggest the involvement of insulin/FOXO1 signaling in the regulation of AQP9 expression.

Chromatin consists of repeating units of nucleosomes, which consist of 146 bp DNA wrapped around an octamer of four core histone proteins (H3, H4, H2A and H2B) (14). Chromatin remodeling is pivotal in regulating gene expression, 
which modulates the accessibility of genomic DNA to regulatory transcription machinery proteins. Covalent modifications are important mechanisms contributing to such remodeling, and include the acetylation, phosphorylation and methylation of histone proteins in the nucleosome (15). Insulin has been shown to alter chromatin structure via the promotion of histone $\mathrm{H} 3$ post-translational modifications $(16,17)$. FOXO1 has the ability to initiate and dynamically modulate active chromatin states (18). Therefore, insulin and FOXO1 were hypothesized to regulate AQP9 expression in part through an epigenetic mechanism involving post-translational modifications of histone $\mathrm{H} 3$. To analyze this hypothesis, in the present study, the effects of insulin treatment and FOXO1 overexpression on AQP9 expression levels, and the histone $\mathrm{H} 3$ modifications at the AQP9 gene promoter in HepG2 human hepatocytes, were examined.

\section{Materials and methods}

Cells and reagents. The cells and reagents used in this study were as follows: HepG2 and Hek 293T human cells (Type Culture Collection of Chinese Academy of Sciences, Shanghai, China), fetal bovine serum (FBS; Gibco-BRL, Carlsbad, CA, USA), Dulbecco's modified Eagle's medium (DMEM; Hyclone Laboratories, Inc., Logan, UT, USA), reagents for quantitative polymerase chain reaction (qPCR; Takara Biotechnology Dalian Co., Ltd., Dalian, China), Lipofectamine ${ }^{\mathrm{TM}} 2000$ (Invitrogen Life Technologies, Carlsbad, CA, USA), and polybrene and recombinant human insulin (Sigma-Aldrich, St. Louis, MO, USA). Primary antibodies against FOXO1 (Abcam, Cambridge, UK), AQP9 (Santa Cruz Biotechnology, Santa Cruz, CA, USA) and $\beta$-actin (Beijing Zhongshan Golden Bridge Biotechnology Co., Ltd., Beijing, China) were used for western blotting.

Cell culture and treatment. The HepG2 and 293T cells were cultured in DMEM supplemented with $10 \%$ FBS, $100 \mathrm{U} / \mathrm{ml}$ penicillin and $100 \mu \mathrm{g} / \mathrm{ml}$ streptomycin at $37^{\circ} \mathrm{C}$ in a humidified $5 \% \mathrm{CO}_{2}$ atmosphere. For insulin treatment, HepG2 cells were seeded at a density of $5 \times 10^{4}$ cells $/ \mathrm{ml}$ into 6 -well plates and incubated in complete medium with $10 \%$ FBS for $24 \mathrm{~h}$. At $\sim 50 \%$ confluence, the cells were starved in FBS-free medium for $6 \mathrm{~h}$ followed by stimulation with $500 \mu \mathrm{M}$ insulin for different durations. Following the treatment, the cells were harvested for further analysis.

Plasmid construction. Total RNA was isolated from the HEK293T cells using TRIzol ${ }^{\mathrm{TM}}$ (Invitrogen Life Technologies) according to the manufacturer's instructions and then reverse-transcribed to cDNA. The cDNA sequence encoding full-length human FOXO1 (GenBank no. NM_002015.3) was amplified by PCR. The PCR primers were as follows: Forward, 5'-AAAGCTAGCATGGCCGAGGCGCCTCAG-3' and reverse, 5'-AAAACTAGTTCAGCCTGACACCCAGCTA-3'. The PCR product was cloned into a pWPI vector (Addgene, Cambridge, MA, USA) and the sequence was confirmed by DNA sequencing.

Preparation and transduction of lentiviral particles. For the production of lentiviral particles expressing FOXO1, HEK293T cells were transfected with the expression vector WPI-FOXO1 along with the packaging vectors psPAX2 and pMD (Addgene) using Lipofectamine 2000 (Invitrogen Life Technologies), according to the manufacturer's instructions. Following incubation for $48 \mathrm{~h}$, the medium containing the lentiviral particles was collected and centrifuged, and aliquots were stored at $-80^{\circ} \mathrm{C}$ until further use.

For lentiviral particle transduction, the HepG2 cells were seeded in $60 \mathrm{~mm}$ dishes and $3 \mathrm{ml}$ viral supernatant was added to $2 \mathrm{ml}$ DMEM with $10 \%$ FBS following cell attachment. The cells were infected for $24 \mathrm{~h}$ in the presence of polybrene $(8 \mu \mathrm{g} / \mathrm{ml})$. After $24 \mathrm{~h}$, the cell culture medium was refreshed. Since the pWPI vector expresses green fluorescent protein (GFP), the transfection efficiency was monitored by detecting GFP expression levels by fluorescence microscopy.

Reverse transcription and $q P C R$. Total RNA was isolated from the HepG2 cells following treatment using TRIzol and cDNA was reverse-transcribed from $1 \mu \mathrm{g}$ total RNA sample. qPCR was performed using a SYBR Green PCR Master Mix kit (Takara Biotechnology Dalian Co., Ltd Dalian, China). The PCR primers were as follows: AQP9 forward, 5'-CTCCTGATTATTGTCATTGC-3' and AQP9 reverse, 5'-ATCCACCAGAAGTTGTTT-3'; $\beta$-actin forward, 5'-CCTGGCACCCAGCACAAT-3' and $\beta$-actin reverse, 5'-GCCGATCCACACGGAGTA-3'. The cycling conditions were as follows: Initial denaturation at $95^{\circ} \mathrm{C}$ for $3 \mathrm{~min}, 40$ cycles of denaturation at $95^{\circ} \mathrm{C}$ for $10 \mathrm{sec}$ and annealing at $60^{\circ} \mathrm{C}$ for $30 \mathrm{sec}$. The data were analyzed with CFX96 Manager software (Bio-Rad Laboratories, Munich, Germany). The relative AQP9 mRNA levels were calculated following normalization to $\beta$-actin mRNA levels.

Western blot analysis. Subsequent to treatment, the HepG2 cells were lysed in radioimmunoprecipitation assay buffer containing $25 \mathrm{mM}$ Tris- $\mathrm{HCl}$ ( $\mathrm{pH} 8.0), 1 \%$ Nonidet-P-40, $0.5 \%$ sodium deoxycholate, $0.1 \%$ sodium dodecyl sulfate, $125 \mathrm{mM} \mathrm{NaCl}$ and $1 \%$ phenylmethanesulfonyl fluoride (Sigma-Aldrich) for $30 \mathrm{~min}$ at $4^{\circ} \mathrm{C}$. The total protein was measured using a Bicinchoninic Protein Assay kit (Pierce Biotechnology, Inc., Rockford, IL, USA). Samples of the total protein extracts $(\sim 60 \mu \mathrm{g})$ were separated by $12 \%$ SDS-PAGE and transferred to a polyvinylidene fluoride membrane. The membrane was incubated overnight with primary antibodies at $4^{\circ} \mathrm{C}$ (anti-AQP9, 1:20 dilution; anti-FOXO1, 1:1,000 dilution). Subsequent to washing three times, the membrane was incubated for $1 \mathrm{~h}$ with horseradish peroxidase-conjugated secondary antibodies (1:3,000 dilution; Beijing Zhongshan Golden Bridge Biotechnology Co., Ltd.). The protein expression was visualized using an Enhanced Chemiluminescence Detection kit (Amersham Pharmacia Biotech, Amersham, UK). The relative intensities of the bands were determined by densitometry using Quantity One software (Bio-Rad, Hercules, CA, USA).

Chromatin immunoprecipitation (ChIP)-PCR assay. ChIP experiments were performed using a Magna ChIP A Chromatin Immunoprecipitation kit (Milipore, Billerica, MA, USA) according to the manufacturer's instructions. Briefly, the cells were incubated in $1 \%$ formaldehyde at room temperature for $10 \mathrm{~min}$, followed by incubation for $10 \mathrm{~min}$ in ice-cold lysis buffer containing a mixture of protease inhibitors. The cells were then sonicated 10 times at $1 \mathrm{~min}$ intervals. Sonication yielded 
DNA fragments $\sim 250$ bp in length, as determined by agarose gel electrophoresis. Following centrifugation, the sonicated sample was diluted 1:10 with dilution buffer, and $20 \mu \mathrm{l}$ diluted supernatant served as an input control. The chromatin solution was precleared using Salmon Sperm DNA/Protein A Agarose Slurry (Millipore, Bedford, MA, USA) for $30 \mathrm{~min}$ and incubated overnight at $4^{\circ} \mathrm{C}$ with anti-acetylated histone $\mathrm{H} 3$. The immunoprecipitated complexes were recovered by adding $30 \mu$ l Salmon Sperm DNA/Protein A Agarose Slurry. Following washing, $5 \mathrm{M} \mathrm{NaCl}$ was added to reverse the formaldehyde cross-linking and the pellets were treated with proteinase K. DNA samples were purified using the QIAquick PCR purification kit (Qiagen, Valencia, CA, USA). Immunoprecipitated DNA and input DNA were amplified by qPCR using the SYBR Green PCR Master Mix kit (Takara Biotechnology Dalian Co., Ltd). The primers used to amplify the IRE locus of the $A Q P 9$ promoter were as follows: Forward, 5'-ATTTCGGGTTCTAAGTCGC-3' and reverse, 5'-TTCCTGGAGATGTCTGGTAAG-3'. All assays were performed in triplicate. The percentage enrichment of immunoprecipitated DNA was calculated relative to the input DNA. The ChIP results were normalized to the input DNA and are expressed as the fold enrichment relative to untreated cells (assigned 1-fold).

Statistical analysis. Statistical analyses were conducted using SPSS version 18.0 (SPSS, Inc., Chicago, IL, USA). The results are presented as the means \pm standard deviation. Significance was determined by Student's t-test or one-way analysis of variance with a Student-Newman-Keuls post hoc test. A P $<0.05$ was considered to indicate a statistically significant difference.

\section{Results}

Insulin suppresses AQP9 mRNA expression in a time-dependent manner. Insulin treatment resulted in a significant $(\mathrm{P}<0.05)$ reduction in AQP9 mRNA expression levels in HepG2 cells, as compared with untreated cells (Fig. 1). Furthermore, the reduction occurred in a time-dependent manner, with maximum reduction observed at $12 \mathrm{~h}$.

Effects of insulin on the post-transcriptional modifications of histone $\mathrm{H} 3$ at the $\mathrm{AQP} 9$ promoter. To verify whether insulin-mediated repression of AQP9 expression was associated with alterations in chromatin remodeling, ChIP and PCR analysis was performed to examine the post-translational modifications of histone $\mathrm{H} 3$ at the $A Q P 9$ promoter. The results revealed that treatment with insulin resulted in a marked reduction in the acetylation and phosphorylation of histone $\mathrm{H} 3$ at the IRE of the $A Q P 9$ promoter, initiated at $0.5 \mathrm{~h}$ and $1 \mathrm{~h}$, respectively, and peaking at $3 \mathrm{~h}$ treatment (Fig. 2). By contrast, a significant $(\mathrm{P}<0.05)$ increase was detected in histone $\mathrm{H} 3$ methylation at the IRE locus of the $A Q P 9$ promoter upon exposure to insulin (Fig. 2). However, no evident change in histone $\mathrm{H} 3$ modification was observed at a control site within the second exon of $A Q P 9$ (data not shown). These results indicate that repression of AQP9 expression by insulin is associated with epigenetic modifications at the promoter.

Enforced expression of FOXO1 stimulates AQP9 expression in Hep 2 cells. The effect of FOXO1 overexpression

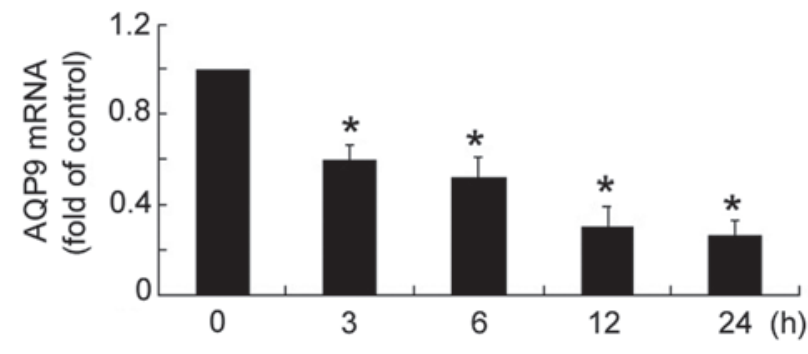

Figure 1. Effects of insulin treatment on AQP9 expression levels. HepG2 human hepatocyte cells were treated with $500 \mu \mathrm{M}$ insulin for different durations and AQP9 mRNA expression levels were determined by quantitative polymerase chain reaction. The data are expressed as the means \pm standard deviation of three independent experiments. ${ }^{*} \mathrm{P}<0.05$ vs. untreated cells $(0 \mathrm{~h})$. AQP9, aquaporin 9; mRNA, messenger RNA.

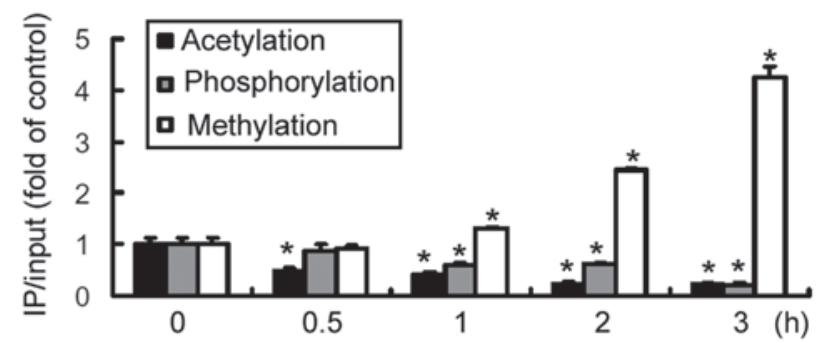

Figure 2. Effects of insulin treatment on the post-translational modifications of histone $\mathrm{H} 3$ at the insulin responsive element (IRE) locus of the aquaporin 9 promoter. HepG2 human hepatocyte cells were treated with $500 \mu \mathrm{M}$ insulin and harvested at 0-3 h for assessment of $\mathrm{H} 3$ acetylation, phosphorylation and methylation at the IRE locus by chromatin immunoprecipitation-polymerase chain reaction. The results are expressed as the fold-enrichment relative to the untreated cells (assigned 1-fold). The error bars indicate the standard deviation of three independent experiments. ${ }^{*} \mathrm{P}<0.05$ vs. untreated cells $(0 \mathrm{~h})$. IP, immunoprecipitation; h, hours.

on AQP9 expression levels was examined. FOXO1-overexpressing plasmid transfection resulted in a $\sim$ three-fold increase in the FOXO1 mRNA and protein expression levels in HepG2 cells, as compared with those of the control cells $(\mathrm{P}<0.05$; Fig. 3A and B). Notably, ectopic expression of FOXO1 significantly $(\mathrm{P}<0.05)$ increased the abundance of AQP9 mRNA in HepG2 cells, as compared with the non-transfected cells (Fig. 4A). The induction of AQP9 by FOXO1 overexpression was further confirmed at the protein level by western blotting $(\mathrm{P}<0.05$; Fig. 4B).

FOXO1 promotes post-transcriptional modifications of histone $\mathrm{H3}$ at the AQP9 promoter. The impact of FOXO1 on epigenetic modifications of the $A Q P 9$ promoter was analyzed. As shown in Fig. 5, the levels of histone H3 acetylation and phosphorylation at the IRE of the $A Q P 9$ promoter was significantly $(\mathrm{P}<0.05)$ elevated in the FOXO1-overexpressing cells, as compared with the control cells. By contrast, the level of histone $\mathrm{H} 3$ methylation at this locus was significantly reduced by FOXO1 overexpression ( $\mathrm{P}<0.05$; Fig. 5).

\section{Discussion}

Glycerol is a predominant substrate in hepatic gluconeogenesis, and the efflux of lipolytic glycerol between adipocytes 
A

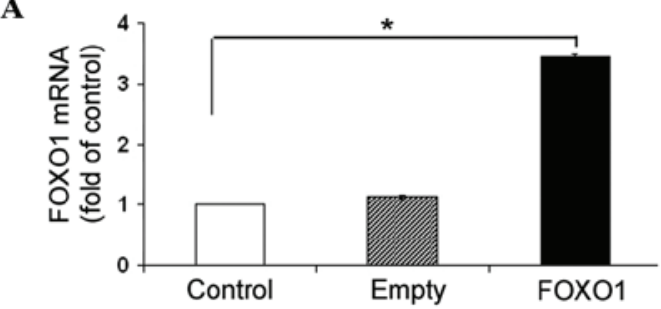

B

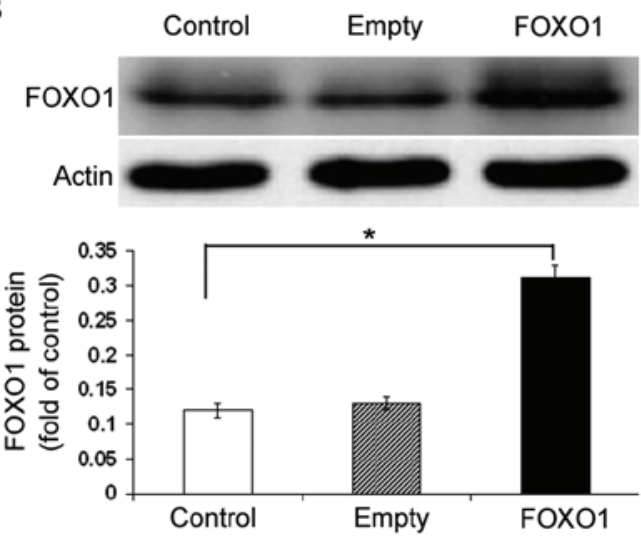

Figure 3. Lentiviral overexpression of FOXO1 in HepG2 human hepatocyte cells. The cells remained unchanged (Control), or were transduced with FOXO1-overexpressing or empty lentiviral particles. After $48 \mathrm{~h}$, the expression levels of FOXO1 protein were determined. (A) Quantitative polymerase chain reaction analysis of FOXO1 mRNA levels. (B) Western blot analysis of FOXO1 protein levels. The western blot data is representative of three independent experiments. The bar graph shows the densitometric quantification of the western blots. ${ }^{*} \mathrm{P}<0.05$ vs. untreated cells. $\mathrm{AQP} 9$, aquaporin 9; FOXO1, forkhead box protein O1; mRNA, messenger RNA.

and the liver is important in modulating lipid and glucose homeostasis. The liver-specific expression of AQP9 facilitates glycerol influx into hepatocytes, and dysregulation of AQP9 is associated with the development of metabolic syndrome (3). Numerous studies have shown that AQP9 expression is negatively regulated in response to insulin (6-8). The data from the present study confirmed the suppression of AQP9 expression in hepatocytes by insulin. Furthermore, this suppression occurred in a time-dependent manner, initiated at $3 \mathrm{~h}$ and reaching a peak after $24 \mathrm{~h}$ treatment. Kuriyama et al (6) identified a consensus IRE in the $A Q P 9$ gene promoter, which may be relevant to the downregulation of AQP9 by insulin. Similarly, insulin has been found to regulate the expression of various target genes via IRE-dependent mechanisms $(19,20)$. Ge et al (20) reported that insulin stimulates the transcription of human acyl-coenzyme A:cholesterol acyltransferase 1 (ACAT1) through an interaction of the functional IRE upstream of the ACAT1 P1 promoter with the CCAAT/enhancer-binding protein $\alpha(\mathrm{C} / \mathrm{EBP} \alpha)$.

The present study provides, to the best of our knowledge, the first evidence that insulin alters the post-transcriptional modifications of histone $\mathrm{H} 3$ at the IRE locus of the $A Q P 9$ promoter, which signifies another mechanism of downregulation of $\mathrm{AQP} 9$ expression by insulin. Numerous reversible histone covalent modifications, including acetylation, phosphorylation and methylation, have been associated with distinct transcription states (21). Histone H3 hyperacetylation is commonly
A

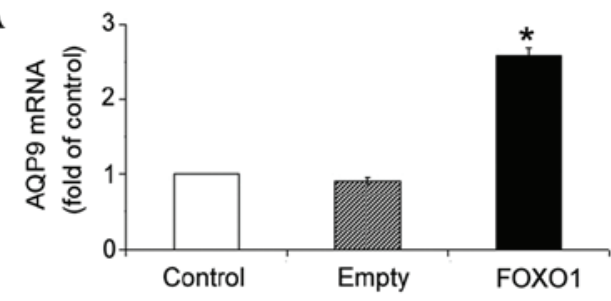

B
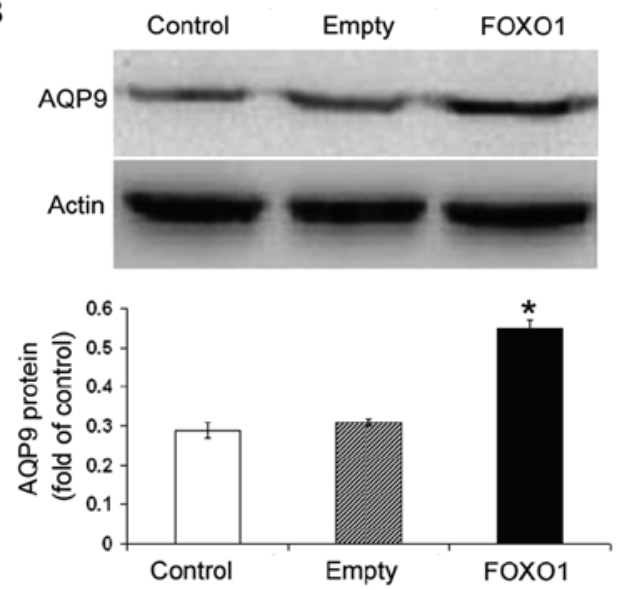

Figure 4. Effects of FOXO1 overexpression on AQP9 expression levels in HepG2 human hepatocyte cells. The cells remained unchanged (Control), or were transduced with FOXO1-overexpressing or empty lentiviral particles and 48 h later, AQP9 expression levels were measured. (A) Quantitative polymerase chain reaction analysis of AQP9 mRNA expression levels. (B) Western blot analysis of AQP9 protein expression levels. Representative blots of three independent experiments are shown in the top panels. The bar graphs show the densitometric quantification of the blots. ${ }^{*} \mathrm{P}<0.05$ vs. untreated cells. AQP9, aquaporin 9; FOXO1, forkhead box protein O1; mRNA, messenger RNA.

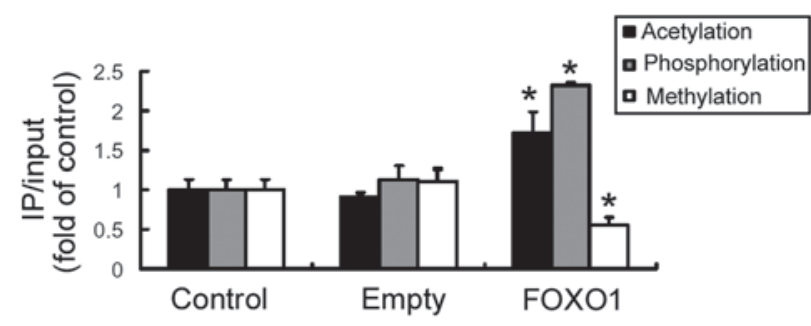

Figure 5. Effects of enforced FOXO1 expression on post-translational modifications of histone $\mathrm{H} 3$ at the insulin responsive element (IRE) locus of the aquaporin $9(A Q P 9)$ promoter. HepG2 human hepatocyte cells remained unchanged (control), or were transduced with FOXO1-overexpressing or empty lentiviral particles and then subjected to the assessment of $\mathrm{H} 3$ acetylation, phosphorylation and methylation at the IRE locus, using chromatin immunoprecipitation-polymerase chain reaction assay. Results are expressed as fold enrichment relative to control cells (assigned 1-fold). Error bars indicate the $\mathrm{SD}$ of three independent experiments. ${ }^{*} \mathrm{P}<0.05$ vs. control. FOXO1, forkhead box protein $\mathrm{O} 1$; mRNA, messenger RNA; IP, immunoprecipitation.

associated with the alleviation of repressive histone-DNA interactions, facilitating the transcription process. By contrast, $\mathrm{H} 3$ methylation at lysine 9 is generally associated with the assembly of compact or closed chromatin surrounding the DNA, resulting in gene silencing. These histone modifications, individually and together, can modulate chromatin structure and gene expression (22). Cheung et al (23) reported that epidermal 
growth factor-induced $\mathrm{H} 3$ phosphorylation affects subsequent acetylation reactions in mammalian cells. The results from the present study demonstrated that the levels of $\mathrm{H} 3$ acetylation and phosphorylation at the IRE locus were significantly reduced 0.5 and $1 \mathrm{~h}$ after insulin treatment, respectively. Similar to H3 phosphorylation, insulin-induced $\mathrm{H} 3$ methylation occurred $1 \mathrm{~h}$ after treatment. These results suggest that insulin treatment resulted in sequential deacetylation and dephosphorylation/methylation of H3, which may cooperatively establish a repressive chromatin configuration.

Numerous transcription factors, such as $\mathrm{C} / \mathrm{EBP} \alpha$, specificity protein 1 , activator protein 1 and FOXO1, have been shown to mediate target gene expression following the administration of insulin $(19,20)$. The present study revealed that the enforced expression of FOXO1 resulted in a significant elevation in AQP9 expression levels in HepG2 cells. Furthermore, FOXO1 overexpression induced $\mathrm{H} 3$ acetylation and phosphorylation, and reduced $\mathrm{H} 3$ methylation in the IRE locus of the $A Q P 9$ promoter, suggesting the formation of a permissive chromatin structure. These results are contrary to the effects of insulin on AQP9 expression and local histone modifications. In addition, the induction of active chromatin states by FOXO1 has been described in a previous study (18). Insulin has the ability to negatively regulate the expression and transcriptional activity of FOXO1 $(24,25)$. FOXO1 has been reported to confer an inhibitory effect of insulin on gene transcription through binding to the IRE in the promoter sequences of target genes (26). Taken together, these data suggest that insulin induces $\mathrm{H} 3$ modifications at the promoter and represses the transcription of AQP9 gene in hepatocytes, which is mediated, at least in part, via FOXO1-dependent alteration of chromatin structure at the IRE locus. Further studies are required to clarify to what extent insulin action is dependent on FOXO1-induced epigenetic modifications. Additionally, the signaling pathways involved in the regulation of FOXO1 by insulin requires further elucidation.

In conclusion, the present study demonstrated that insulin-induced transcriptional repression of AQP9 gene expression in hepatocytes is associated with FOXO1-mediated $\mathrm{H} 3$ modifications at the IRE locus of the $A Q P 9$ promoter. These findings warrant further investigation of the clinical significance of epigenetic regulation of AQP9 expression in treating metabolic syndrome.

\section{References}

1. Verkman AS: Aquaporins. Curr Biol 23: R52-R55, 2013.

2. Buffoli B: Aquaporin biology and nervous system. Curr Neuropharmacol 8: 97-104, 2010.

3. Maeda N: Implications of aquaglyceroporins 7 and 9 in glycerol metabolism and metabolic syndrome. Mol Aspects Med 33: 665-675, 2012

4. Rojek AM, Skowronski MT, Füchtbauer EM, et al: Defective glycerol metabolism in aquaporin 9 (AQP9) knockout mice. Proc Natl Acad Sci USA 104: 3609-3614, 2007.

5. Jelen S, Wacker S, Aponte-Santamaría C, et al: Aquaporin-9 protein is the primary route of hepatocyte glycerol uptake for glycerol gluconeogenesis in mice. J Biol Chem 286: 44319-44325, 2011.
6. Kuriyama H, Shimomura I, Kishida K, et al: Coordinated regulation of fat-specific and liver-specific glycerol channels, aquaporin adipose and aquaporin 9. Diabetes 51: 2915-2921, 2002.

7. Castro Parodi M, Farina M, Dietrich V, Abán C, Szpilbarg N Zotta E and Damiano AE: Evidence for insulin-mediated control of AQP9 expression in human placenta. Placenta 32: 1050-1056, 2011.

8. Rodríguez A, Catalán V, Gómez-Ambrosi J, et al: Insulinand leptin-mediated control of aquaglyceroporins in human adipocytes and hepatocytes is mediated via the PI3K/Akt/mTOR signaling cascade. J Clin Endocrinol Metab 96: E586-E597, 2011.

9. Carbrey JM, Gorelick-Feldman DA, Kozono D, Praetorius J, Nielsen S and Agre P: Aquaglyceroporin AQP9: solute permeation and metabolic control of expression in liver. Proc Natl Acad Sci USA 100: 2945-2950, 2003.

10. Kamagate A, Qu S, Perdomo G, et al: FoxO1 mediates insulin-dependent regulation of hepatic VLDL production in mice. J Clin Invest 118: 2347-2364, 2008.

11. Matsumoto M, Pocai A, Rossetti L, Depinho RA and Accili D: Impaired regulation of hepatic glucose production in mice lacking the forkhead transcription factor Foxol in liver. Cell Metab 6: 208-216, 2007.

12. Tsuchida A, Yamauchi T, Ito Y, et al: Insulin/Foxol pathway regulates expression levels of adiponectin receptors and adiponectin sensitivity. J Biol Chem 279: 30817-30822, 2004.

13. Xiao X, Mei ZC, Qiu LW, et al: Effect of forkhead transcription factor 1 gene silencing on expression of aquaporin 9 in normal human liver cells. Chin J Biologicals 10: 1157-1161, 2011.

14. Sharma S, Kelly TK and Jones PA: Epigenetics in cancer. Carcinogenesis 31: 27-36, 2010.

15. Gräff J, Kim D, Dobbin MM and Tsai LH: Epigenetic regulation of gene expression in physiological and pathological brain processes. Physiol Rev 91: 603-649, 2011.

16. Kabra DG, Gupta J and Tikoo K: Insulin induced alteration in post-translational modifications of histone $\mathrm{H} 3$ under a hyperglycemic condition in L6 skeletal muscle myoblasts. Biochim Biophys Acta 1792: 574-583, 2009.

17. Gupta J and Tikoo K: Involvement of insulin-induced reversible chromatin remodeling in altering the expression of oxidative stress-responsive genes under hyperglycemia in 3T3-L1 preadipocytes. Gene 504: 181-191, 2012.

18. Hatta M and Cirillo LA: Chromatin opening and stable perturbation of core histone: DNA contacts by FoxO1. J Biol Chem 282: 35583-35593, 2007.

19. Ornskov D, Nexo E and Sorensen BS: Insulin induces a transcriptional activation of epiregulin, HB-EGF and amphiregulin, by a PI3K-dependent mechanism: identification of a specific insulin-responsive promoter element. Biochem Biophys Res Commun 354: 885-891, 2007.

20. Ge J, Zhai W, Cheng B, et al: Insulin induces human acyl-coenzyme A: cholesterol acyltransferasel gene expression via MAP kinases and CCAAT/enhancer-binding protein $\alpha$. J Cell Biochem 114: 2188-2198, 2013.

21. Yan C and Boyd DD: Histone H3 acetylation and H3 K4 methylation define distinct chromatin regions permissive for transgene expression. Mol Cell Biol 26: 6357-6371, 2006.

22. Fischle W, Wang YM and Allis CD: Histone and chromatin cross-talk. Curr Opin Cell Biol 15: 172-183, 2003.

23. Cheung P, Tanner KG, Cheung WL, Sassone-Corsi P, Denu JM and Allis CD: Synergistic coupling of histone H3 phosphorylation and acetylation in response to epidermal growth factor stimulation. Mol Cell 5: 905-915, 2000.

24. Matsuzaki H, Daitoku H, Hatta M, Tanaka K and Fukamizu A: Insulin-induced phosphorylation of FKHR (Foxol) targets to proteasomal degradation. Proc Natl Acad Sci USA 100: 11285-11290, 2003.

25. Miao H,Zhang Y, Lu Z, Liu Q and Gan L: FOXO1 involvement in insulin resistance-related pro-inflammatory cytokine production in hepatocytes. Inflamm Res 61: 349-358, 2012.

26. Durham SK, Suwanichkul A, Scheimann AO, Yee D, Jackson JG, Barr FG and Powell DR: FKHR binds the insulin response element in the insulin-like growth factor binding protein-1 promoter. Endocrinology 140: 3140-3146, 1999. 